\title{
GENERATION OF SINGLE AND DOUBLE PULSES USING A SYMMETRICAL MACH-ZEHNDER INTERFEROMETER EMPLOYING MICROSTRUCTURED CHALCOGENIDE FIBRES: NUMERICAL ANALYSIS
}

\author{
M.-H. Jin, S.-H. Yang, and J.-W. Wu \\ School of Physics and Electronic Engineering, Chongqing Normal University, Chongqing 401331, People's Republic of China \\ Email: jwwu@cqnu.edu.cn
}

Received 9 May 2018; revised 7 October 2018; accepted 15 October 2018

\begin{abstract}
In this numerical work, a Mach-Zehnder interferometer device with sample and reference arms composed of microstructured chalcogenide optical fibres with $\mathrm{AsSe}_{2}$ core and $\mathrm{As}_{2} \mathrm{~S}_{5}$ cladding is presented to generate single and double pulses. An unchirped hyperbolic secant-shaped pulse with enough high peak power centered at $1958 \mathrm{~nm}$ wavelength and a continuous wave with a very weak power level at $2070 \mathrm{~nm}$ wavelength are simultaneously introduced into the sample arm, in which stimulated Raman scattering and strong cross-phase modulation induced by two co-propagating waves give rise to a significant phase shift for a continuous wave. In the reference arm, the continuous wave directly passes through the fibre, where the transmitted wave has a slight phase shift due to the influence of self-phase modulation. Therefore, at the output port of the configuration, the re-combined optical fields at $2070 \mathrm{~nm}$ wavelength have a significant phase difference. As a result, both single and double pulses caused by the optical interference at the output port are achieved by judiciously adjusting the phase difference which is mainly controlled by the peak power and temporal width of the input optical pulse, as well as the length of a microstructured optical fibre. In addition, both widths of the single pulse and spacing of the double pulses are directly dependent on the temporal width of the input pulse and the fibre length of the arm.
\end{abstract}

Keywords: nonlinear optics, microstructured optical fibre, Mach-Zehnder interferometer, pulse generation

PACS: 42.15.Eq, 42.81.Qb, 42.65.Re

\section{Introduction}

The Mach-Zehnder interferometer (MZI) as a classical optoelectronic device has extensively been presented to perform various optical functions such as wavelength conversion [1], logic gates [2], optical switching [3], optical delay [4], sensor [5], amplifier [6] and microwave filter [7]]. Generally, in order to compact the setup and enhance the nonlinear behaviours of transmission lines, some highly nonlinear elements including a semiconductor optical amplifier (SOA) [8], silicon [9], plasmonic [10] as well as photonic crystals [11, 12], etc. are implanted into the arms of the MZI configuration. Therefore, various kinds of highly nonlinear materials assisted MZI devices have been intensively studied to improve the working performance in recent reports, in which one can see that the main aim of MZI is to induce the phase shift of a propagating wave in the arm line, resulting in the effective interference effect for the recombined waves owing to their phase difference at the output port. Here, it is shown that the presented MZI devices are well compacted owing to the applications of various kinds of highly nonlinear optical materials.

In the family of highly nonlinear elements, the $\mathrm{AsSe}_{2}$ core microstructured optical fibre (MOF) 
has been proposed and demonstrated to achieve supercontinuum (SC) generation [13-16], and stimulated Raman scattering (SRS) due to its strong nonlinear coefficient that is about three orders of magnitude higher than that of a conventional glass fibre [17]. In addition, compared to other cases in semiconductor materials, e.g. SOA and silicon, the influences of free carriers and nonlinear photon absorptions on the transmission waves are not applicable for the $\mathrm{AsSe}_{2}$ core MOF which also has a strong nonlinear coefficient compared to that of the conventional optical fibre. Obviously, the $\mathrm{AsSe}_{2}$ core MOF is very advantageous to compact the optical device and realize various functions.

Hence, in order to further explore and present the MOF related applications, the $\mathrm{AsSe}_{2}$ core $\mathrm{MOF}$ based MZI configuration is proposed to produce single and double pulses in this work, in which the highly nonlinear MOFs as phase shift lines are implanted into the two arms of MZI. The optical properties including the temporal width, pulses spacing and frequency chirp of the outcome pulse are directly dependent on the length of MOF, the temporal width and peak power of the input optical pulse. In reality, the techniques of pulse generations are very interesting subjects, according to Refs. [18-24], in which various interference devices, e.g. MZI configurations, have been utilized to obtain new optical pulses. From these reports, one can find that the achieved results have obvious competition abilities and advantages for generating pulses. However, compared to these attained results, the method of pulse generation presented in this study has outstanding advan- tages such as a compact structure, negligible carrier effect, symmetrical shape, controllable pulse width and spacing, as well as flexible operation. In addition, the generated pulse can also be centered at a different wavelength by changing the wavelength of the input continuous wave. In this study, the pulse generator employing the $\mathrm{AsSe}_{2}$ core MOF based MZI device is shown to have some potential applications, e.g. the measurement of indirectly modulated terahertz $(\mathrm{THz})$ pulse [25] and harmonic generation [26].

\section{Device and theoretical framework}

The setup of the single and double pulses generator based on the MZI structure, employing the MOF with a $2.6 \mu \mathrm{m}$ core diameter in two arms, is illustrated in Fig. 1, in which the working principle is given as follows. Firstly, the initial continuous wave labelled as ' $B_{0}$ ' which is centered at the wavelength $\lambda_{B}$ is equally divided into two parts $\left(B_{1}, B_{2}\right)$ by the fibre coupler $C_{1}$ with the $50 / 50$ power splitting ratio. The continuous wave $B_{1}$ directly passes through the reference arm, in which its nonlinear phase shift mainly caused by selfphase modulation (SPM) is very slight, resulting from the low power level for the input continuous wave. It should be pointed out that other nonlinear effects, e.g. SRS, can also be ignored as a result of low power for the propagating wave. Another part $B_{2}$ of the continuous wave is entered into the sample arm after it passes through the coupler $C_{2}$, having the $50 / 50$ power splitting ratio. However, in order to enhance the nonlinear phase shift of the propagating beam at wavelength $\lambda_{B}$,

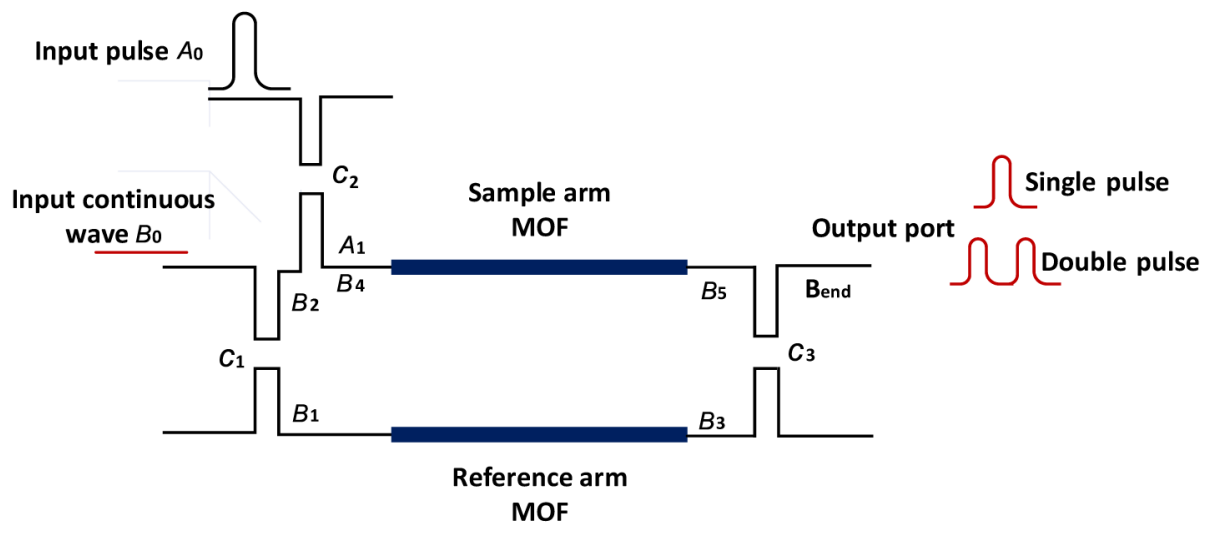

Fig. 1. A schematic diagram of the MOF based MZI configuration for generation of single and double pulses. 
an input pulse labelled as ' $A_{0}$ ' centered at $\lambda_{A}$ is also launched into the sample arm via the coupler $C_{2}$, in which the split pulse is labelled as $A_{1}$. In the sample arm, both the pulse and continuous wave are co-propagating along the MOF line, in which because of the combined influences of dispersion, SPM, cross-phase modulation (XPM) and SRS, the total phase shift of the continuous wave can be rapidly increased. Finally, the output beams from the sample arm and the reference arm are, again, recombined through the coupler $C_{3}$ whose power splitting ratio is also equal to $50 / 50$. As a consequence, the recombined waves centered at wavelength $\lambda_{B}$ produce a strong interference effect owing to their significant phase difference $\Delta \Phi$. Here, only one output port of the MZI device is selected as the output port, as shown in Fig. 1. Therefore, according to the interference mechanisms, the outcome power is enhanced for the constructive interference, i.e. $\Delta \Phi=2 \mathrm{k} \pi(k=0$, $1,2 \ldots)$, and the output power is equal to zero as a result of the fully destructive interference, i.e. $\Delta \Phi=(2 \mathrm{k}+1) \pi(k=0,1,2 \ldots)$. Therefore, by properly adjusting the phase difference between the recombined two waves at $\lambda_{B}$, both the single pulse and the double pulses can be achieved, respectively.

Through the couplers $C_{1}$ and $C_{2}$, the output amplitudes $B_{1}$ and $B_{4}$ for the continuous wave $B_{0}$ and the splitting amplitude $A_{1}$ for the input pulse $A_{0}$ are described as

$$
\begin{aligned}
& B_{1}=\mathrm{i} \sqrt{1-a_{1}} B_{0}, \\
& B_{4}=\sqrt{a_{1}} \sqrt{a_{2}} B_{0}, \\
& A_{1}=\mathrm{i} \sqrt{1-a_{2}} A_{0} .
\end{aligned}
$$

Here, $a_{1}(=0.5)$ and $a_{2}(=0.5)$ are the power splitting ratios of couplers $C_{1}$ and $C_{2}$, respectively. After the amplitudes $B_{1}, B_{4}$ as well as $A_{1}$ are, respectively, launched into the MOF lines, the evolutions of the pulse and the continuous wave in the sample arm can be, respectively, described by the corresponding transmission equations, written as

$$
\begin{aligned}
& \frac{\partial A}{\partial z}+d \frac{\partial A}{\partial T}+\frac{\mathrm{i} \beta_{2 A}}{2} \frac{\partial^{2} A}{\partial T^{2}} \\
& =\mathrm{i} \gamma_{A}\left[|A|^{2}+\left(2-f_{R}\right)|B|^{2}\right] A-\frac{g_{A}}{2}|B|^{2} A,
\end{aligned}
$$

$$
\begin{aligned}
& \frac{\partial B}{\partial z}+\frac{\mathrm{i} \beta_{2 B}}{2} \frac{\partial^{2} B}{\partial T^{2}}=\mathrm{i} \gamma_{B}\left[|B|^{2}\right. \\
& \left.+\left(2-f_{R}\right)|A|^{2}\right] B+\frac{g_{B}}{2}|A|^{2} B .
\end{aligned}
$$

Here, both $A$ and $B$ are the amplitudes of the propagating pulse and the continuous wave, respectively. $z$ is the distance of propagation, $T=t-z / v_{\mathrm{g} B}$ is the retarded time-frame, $d=$ $1 / v_{\mathrm{g} A}-1 / v_{\mathrm{g} B}$ is the walk-off parameter resulting from the group-velocity mismatch between two propagating beams, and $v_{\mathrm{g} A}$ and $v_{\mathrm{g} B}$ are the group velocities of wave $A$ and wave $B$, respectively. In this case, $v_{\mathrm{g} A}$ is smaller than $v_{\mathrm{g} B}$. $\beta_{2}$ represents the coefficient of the group velocity dispersion. $\gamma$ is the nonlinear coefficient, and $f_{\mathrm{R}}$ denotes the fractional Raman contribution. $g_{A}$ and $g_{B}$ are the Raman-gain coefficient at $\lambda_{A}$ and $\lambda_{B}$, respectively. It should be pointed out that the linear loss in the MOF is not considered due to the relatively short MOF in the calculation. In the reference arm, only self-phase modulation will produce a weak influence on the propagating wave $B$ with a weak power level. Obviously, at the end of the MOF of the sample arm and the reference arm, the required output optical fields labelled as $B_{5}$ and $B_{3}$ can be obtained by numerically calculating the corresponding transmission equations. Through the coupler $C_{3}$ with the power splitting ratio, $a_{3}(=0.5)$, the outcome optical field $B_{\text {end }}$ in the output port illustrated in Fig. 1 is written as

$$
B_{\text {end }}=\sqrt{a_{3}} B_{5}+\mathrm{i} \sqrt{1-a_{3}} B_{3} .
$$

Here, it is shown that the achieved outcome field $B_{\text {end }}$ is the direct result of the interference of $\sqrt{a_{3}} B_{5}$ and $\mathrm{i} \sqrt{1-a_{3}} B_{3}$. Therefore, by properly controlling the phase difference of recombined optical fields, both the single and the double pulses can be generated in the output port of the MOF based MZI. In order to perform the numerical calculation, the adopted parameters are given in Table 1. Here, it should be pointed out that, based on the physical mechanism of pulse generation, one can still perform the function of pulse generation while both of two optical fields are counter-propagating along the sample arm. But, the corresponding results are not discussed in this study. 
Table 1. Values of numerical calculation

\begin{tabular}{cc}
\hline Parameter & Value \\
\hline$\lambda_{A}(\mathrm{~nm})$ & 1958 \\
\hline$\lambda_{B}(\mathrm{~nm})$ & 2070 \\
\hline$\beta_{2 A}\left(\mathrm{ps}^{2} / \mathrm{m}\right)$ & 0.92 \\
\hline$\beta_{2 B}\left(\mathrm{ps}^{2} / \mathrm{m}\right)$ & 0.45 \\
\hline$d(\mathrm{ps} / \mathrm{m})$ & 48.1 \\
\hline$\gamma_{A}\left(\mathrm{~km}^{-1} \mathrm{~W}^{-1}\right)$ & 6649 \\
\hline$\gamma_{B}\left(\mathrm{~km}^{-1} \mathrm{~W}^{-1}\right)$ & 6289 \\
\hline$f_{\mathrm{R}}$ & 0.148 \\
\hline$g_{A}(\mathrm{~m} / \mathrm{W})$ & $1.53 \times 10^{-11}$ \\
\hline$g_{B}(\mathrm{~m} / \mathrm{W})$ & $1.45 \times 10^{-11}$ \\
\hline
\end{tabular}

\section{Results and discussion}

In the cases of various MOF lengths, $z$, the temporal shapes of outcome pulses, the phase differences of the recombined waves at wavelength $\lambda_{B}$ and the frequency chirps are, respectively, shown in Fig. 2(a, b, c), in which 100, 150 and $300 \mathrm{~mm}$ long MOFs are adopted to display various output pulses. An unchirped hyperbolic secant-shaped pulse with $5.5 \mathrm{~W}$ peak power is selected as the ini- tial pulse, and its temporal width, $T_{A 0}$, is equal to 5 ps at $1 /$ e intensity point, i.e. corresponding to the $\sim 8.8$ ps full width at half maximum (FWHM). The initial continuous wave has the $1 \mathrm{~mW}$ power level that is very weak compared to the peak power of the initial input pulse. As a consequence, the phase shift of the wave $B$ in the sample arm is mainly contributed by the XPM between two co-propagating waves, and the influences of SPM and SRS on the phase shift of the wave $B$ are very slight because of a weak power level for the wave $B$ and an improper wavelength spacing for performing the SRS process of two propagating beams. As such, the SPM induced phase shift for the wave $B$ is also small in the reference arm. In this case, the MOF length in the sample arm is identical to that of the reference arm so that the remarkable phase difference, shown in Fig. 2(b), for the recombined wave $B$ at the end of the MZI device is increased to an expectant level owing to the strong nonlinear interaction such as the XPM of two optical fields in the sample arm. The main goal is to achieve the single or the double pulses,
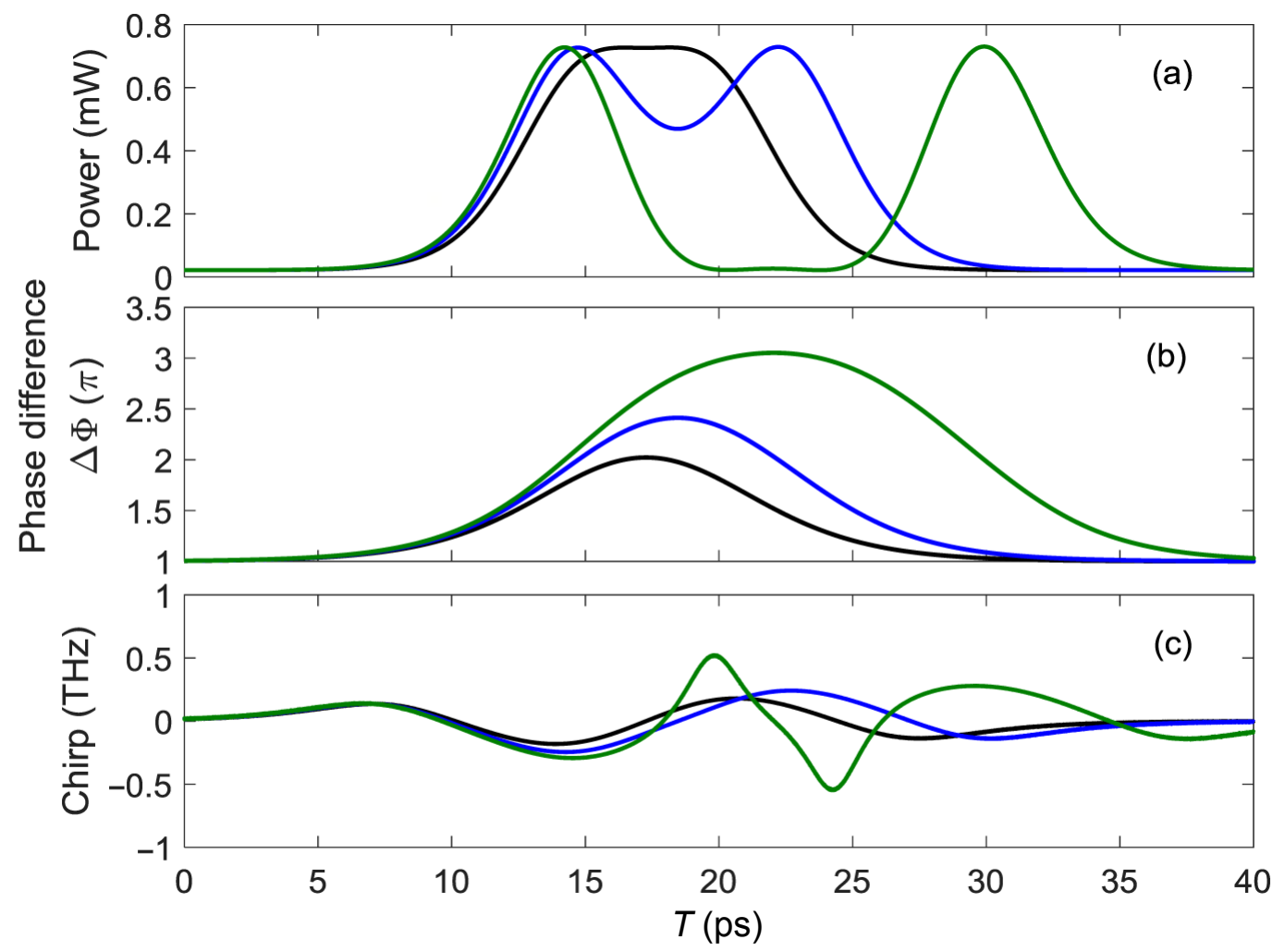

Fig. 2. Temporal shapes (a), phase differences (b) and chirps with various MOF lengths (c). Black lines with $100 \mathrm{~mm}$ long MOF, blue lines with $150 \mathrm{~mm}$ long MOF and green lines with $300 \mathrm{~mm}$ long MOF. 
illustrated in Fig. 2(a). In the case of a 100-mm long MOF line, the maximum phase difference shown in Fig. 2(b) is equal to $\sim 2 \pi$ that induces the fully constructive interference, resulting in the generation of the single pulse with 10 ps FWHM. For the case of the $150 \mathrm{~mm}$ long MOF, the maximum phase difference is also increased to $\sim 2.4 \pi$ that leads to a slight reduction for output power in the central part of the pulse, resulting from the weak destructive interference. Therefore, the achieved pulse with FWHM extended to $\sim 12.8$ ps is characterized by two peaks, i.e. a dip structure in the pulse profile. Because of the effect of group velocity mismatch, the range of temporal overlap for the case of the $100 \mathrm{~mm}$ long MOF is shorter than that for the case of the $150 \mathrm{~mm}$ long MOF so that the outcome pulse can have a wider time duration by properly extending the MOF length. In addition, it is known that, under a fixed peak power for the input pulse $A_{0}$ condition, the maximum phase difference of the wave $B$ is proportional to the MOF length, namely, the maximum phase difference for the wave $B$ approaches $\sim 3 \pi$ in the case of the $300 \mathrm{~mm}$ MOF. As a result, the dip of the output pulse with two peaks is further decreased to the zero power level attributed to the fully destructive interference so that the perfect double pulses will be displayed in the output port of the MZI configuration. Here, the FWHM of the left pulse and the right pulse are, respectively, compressed to $\sim 5$ and 5.5 ps, which are less than that of the input pulse $A_{0}$. In order to characterize the output pulse, Fig. 2(c) plots their corresponding frequency chirps that take on the nonlinear process across the total pulse. The absolute maximum of the frequency chirp is proportional to the MOF length. In the case of the single-pulse output, the central part of the output pulse has a linear up-chirp. However, while the output pulse is evolved into the double pulses, the chirp of the left (right) pulse is less than zero (up to zero). The phenomena can be explained by the cast of the XPM effect between two co-propagating optical fields. It is well known that in the MOF the input pulse with enough high peak power has the SPM induced nonlinear frequency chirp across the total pulse, namely, a negative chirp near the leading edge and a positive chirp near the trailing edge are observed. In the case of the $300 \mathrm{~mm}$ long MOF, the achieved double pulses are generated by nonlinear interaction of the edges of the pulse and the continuous wave. Therefore, because of the influences of the XPM effect on the continuous wave and group-velocity mismatch, one can know that in Fig. 2 the chirp near the leading (trailing) edge of the pulse imposes on the left (right) pulse. In order to clearly display the influence of the MOF length on the properties of the output pulse, Fig. 3 in which other parameters adopted are identical to those of Fig. 2 shows the characteristics including the shape and the frequency chirp of the outcome pulse as a function of the MOF length. From the $3 \mathrm{D}$ view and the aerial view, shown in Fig. $3(a, b)$, the single pulse is achieved in the case of the less than $110 \mathrm{~mm}$ long MOF, in which the peak power of the output pulse is gradually enhanced with the MOF length increase. This is because the corresponding phase difference is proportional to the MOF length, i.e. while the enhanced phase difference is increased to $2 \pi$ by increasing the MOF length, the peak of the single pulse reaches the maximum value. Obviously, after the maximum value of phase difference overruns $2 \pi$, i.e. in the case of the around $155 \mathrm{~mm}$ long MOF, the achieved single pulse acquires a distinct twopeak structure because the maximum phase difference is up to $2 \pi$. As the MOF length is increased from $\sim 210-300 \mathrm{~mm}$, the perfect double pulses with its power between two pulses strongly suppressed are achieved. The behaviour implies that the fully destructive interference between two pulses occurs, resulting from the phase difference of about $3 \pi$, which is attributed to the nonlinear interaction between the peak of the pulse $A$ and the continuous wave. In addition, because of the walk-off effect between two optical fields, one can see that the spacing of two pulses is gradually broadened with the MOF length increase. The nonlinear frequency chirp imposed on the outcome pulse is significantly enhanced by extending the MOF length, as displayed in Fig. 3(c).

Properties such as the pulse evolutions and nonlinear frequency chirp of outcome single and double pulses, as displayed in Fig. 4, are plotted with respect to the initial peak power of the input pulse $A_{0}$, ranging from 1 to $8 \mathrm{~W}$. Here, the MOF length is adjusted to $150 \mathrm{~mm}$, and other parameters are the same as those in Fig. 3. In the sample arm, the phase shift of the wave $B$ is mainly induced by the nonlinear effects such as XPM and SRS that are strongly dependent on the peak power 
(a)
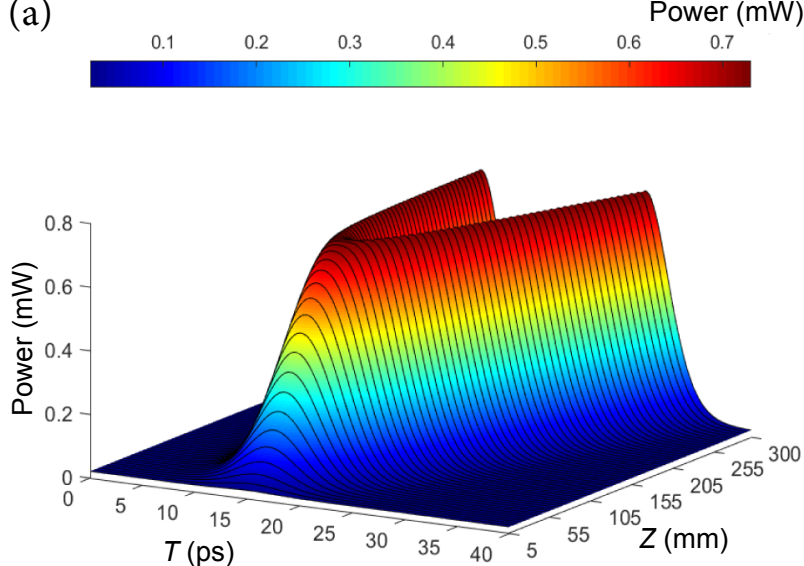

(b)
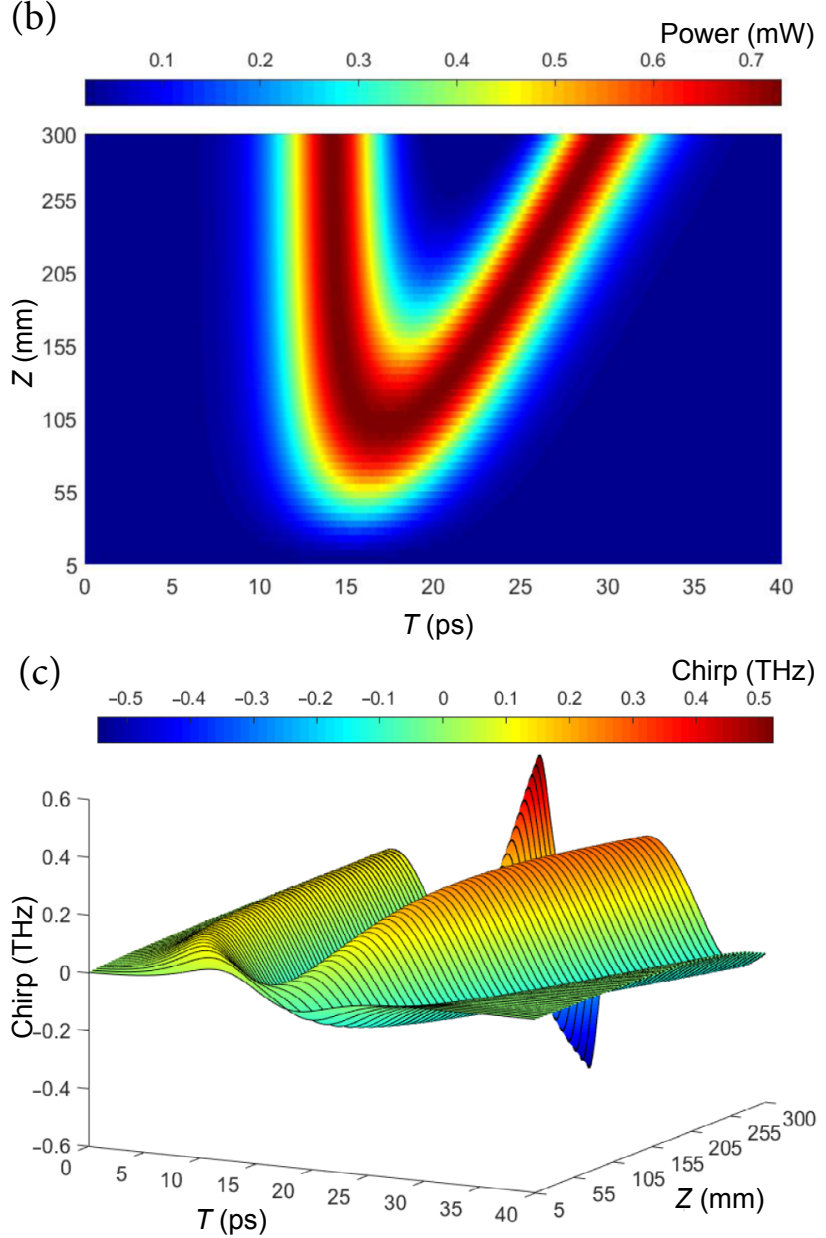

Fig. 3. Temporal 3-D (a) and temporal aerial (b) views, and the chirp of the outcome pulse against the length of MOF (c).

of the co-propagating pulse $A$, i.e. the nonlinear phase shift of the wave $B$ is significantly increased by increasing the initial peak power of the pulse $A_{0}$. As the input power of the pulse $A_{0}$ is increased from 1 to $\sim 4.5 \mathrm{~W}$, the peak of the generated single pulse, shown in Fig. $4(a, b)$, is gradually enhanced owing to the enhanced peak of the pulse $A$ which causes the maximum phase difference between two recombined waves $B$ to approach $2 \pi$. While the initial power of the pulse $A_{0}$ is varied from $\sim 4.5$ to $6.5 \mathrm{~W}$, the output pulse exhibits two remarkable peaks. This behaviour implies that the maximum phase difference is up to $2 \pi$, and much less than $3 \pi$.

(a)
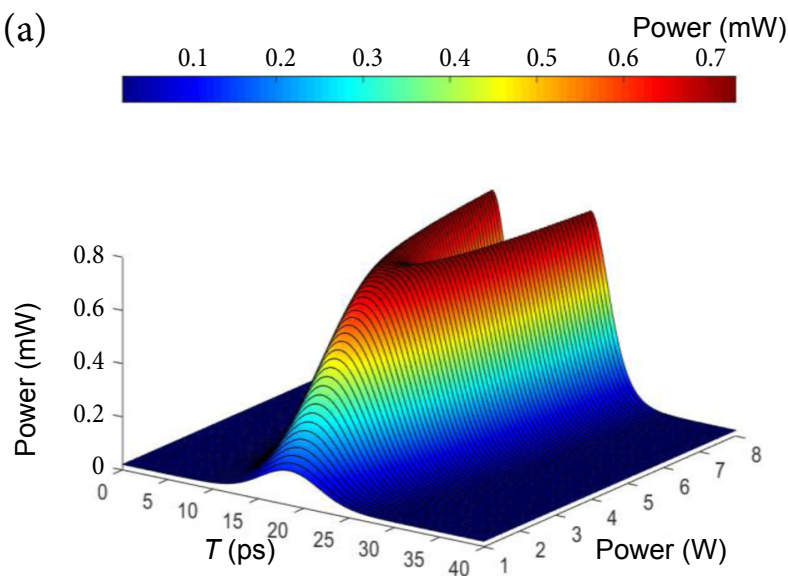

(b)

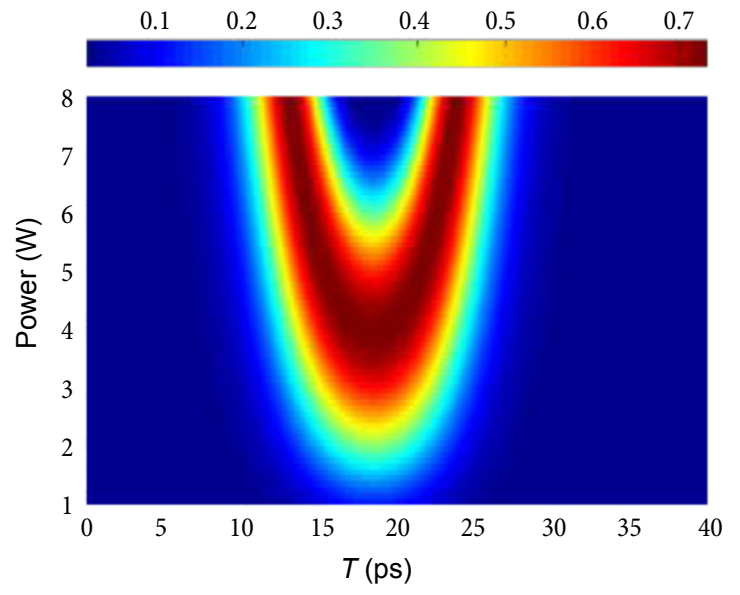

(c)
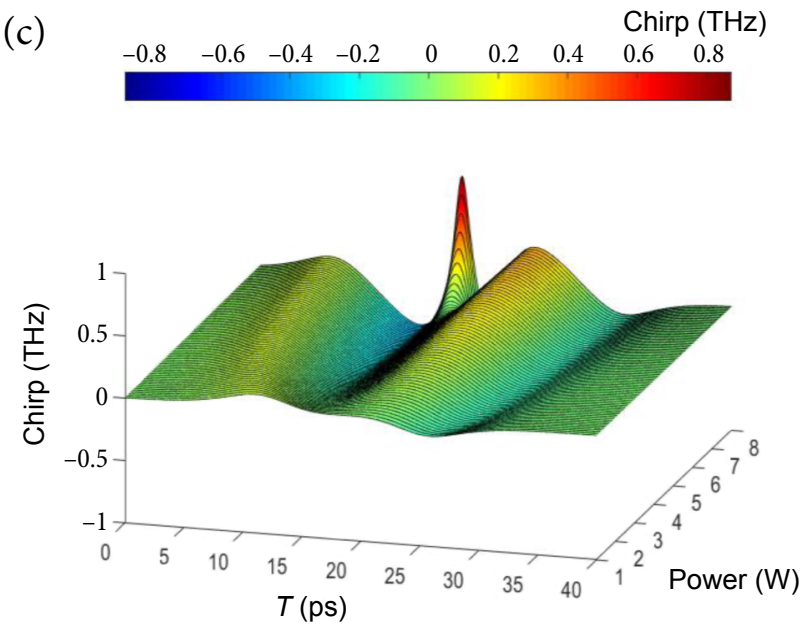

Fig. 4. Temporal 3-D (a) and temporal aerial (b) views, and the chirp of the outcome pulse against the initial peak power of pulse $A_{0}(\mathrm{c})$. 
By adjusting the power of the pulse $A_{0}$ from $\sim 6.5$ to $8 \mathrm{~W}$, the maximum phase difference is equal to around $3 \pi$ that gives rise to the fully destructive interference in the central part of the nonlinearly temporal interaction range between two optical fields. As a consequence, the perfect double pulses are illustrated in Fig. $4(a, b)$, in which it is shown that the spacing of two pulses is slightly changed by increasing the power of the pulse $A_{0}$. Because of the function of pulse compression based on the MZI configuration [27], the temporal widths of the double pulses are significantly shorter than those of the single pulse and the pulse $A_{0}$. The nonlinear frequency chirp of the outcome pulse, as shown in Fig. 4(c), is gradually enhanced with the increase of the pulse $A_{0}$ peak power that results in the enhanced nonlinear interactions between two optical fields such as the XPM effect.

In the cases of various MOF lengths, the shapes of the single pulse that are achieved by properly selecting the initial power of the pulse $A_{0}$ are displayed in Fig. 5, in which the adopted other parameters are not changed compared to those used in calculations. As the MOF length is varied from 50 to $450 \mathrm{~mm}$, the temporal width of the single pulse is significantly broadened due to the walk-off effect between the wave $B$ and the pulse $A$. Namely, the nonlinear temporal interaction range between two optical fields is extended with the MOF length increase. It is known that the corresponding phase difference is directly proportional to the initial peak power of the pulse $A_{0}$ and the MOF length. In order to increase the output peak of the single pulse to be the maximum value, the phase difference between two recombined waves should be close to $2 \pi$ so that a higher peak power for the pulse $A_{0}$ is requested in the case of a shorter MOF length. As can be seen in Fig. 5, the output peak for each single pulse is almost equal to $\sim 0.72 \mathrm{~mW}$ under various MOF length conditions, in which one can see that, in the case of the $50 \mathrm{~mm}$ long $\mathrm{MOF}$, the requested power $(\sim 10.4 \mathrm{~W})$ for the pulse $A_{0}$ is higher than $\sim 2.4 \mathrm{~W}$ at the $450 \mathrm{~mm}$ long MOF, i.e. while MOF lengths are fixed at 50, 150, 250, 350 and $450 \mathrm{~mm}$, the requested power is adjusted to $\sim 10.4,4.0,2.9,2.5$ and $2.4 \mathrm{~W}$, respectively. As the MOF length is increased from 50 to $450 \mathrm{~mm}$, the temporal FWHM for the single pulse is quickly broadened from $\sim 9.8$ to $\sim 23 \mathrm{ps}$, resulting from the walk-off effect between the pulse $A$ and the wave $B$. When the maximum phase difference is close to $3 \pi$, the perfect double pulses plotted in Fig. 6 are attained. The physical mechanism for generating the double pulses is identical to that of the single pulse so that the requested input power for the pulse $A_{0}$ with the $50 \mathrm{~mm}$ long MOF is as high as $\sim 21 \mathrm{~W}$. As the MOF length is increased to $150 \mathrm{~mm}$, the adopted initial peak of the pulse $A_{0}$ is significantly reduced to $\sim 8 \mathrm{~W}$, resulting in the fully destructive interference due to the $\sim 3 \pi$ phase difference. In the case of the $450 \mathrm{~mm}$ long MOF, only $\sim 5 \mathrm{~W}$ initial power can give rise to the perfect double pulse. Here, the peak levels of the double pulses are still equal to $\sim 0.72 \mathrm{~mW}$ for various MOF lengths by judiciously selecting power of the input pulse $A_{0}$. However, the temporal FWHMs of the double pulse are slightly broadened from $\sim 4$ to $\sim 6 \mathrm{ps,} \mathrm{which} \mathrm{are}$ still shorter than that of the input pulse $A_{0}$. In addition, the spacing between two pulses in the double

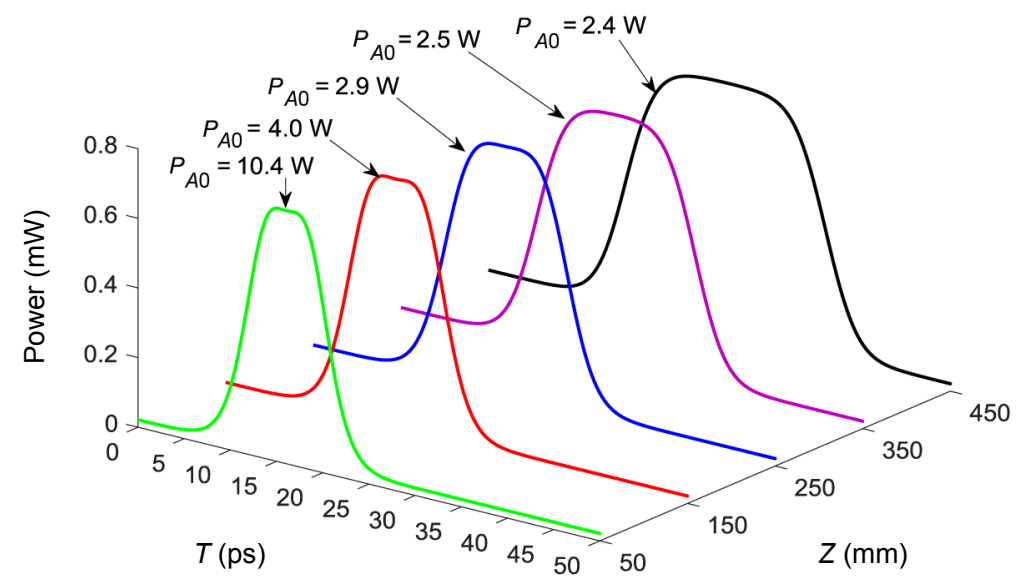

Fig. 5. Temporal shapes of the single pulse with various MOF lengths. 


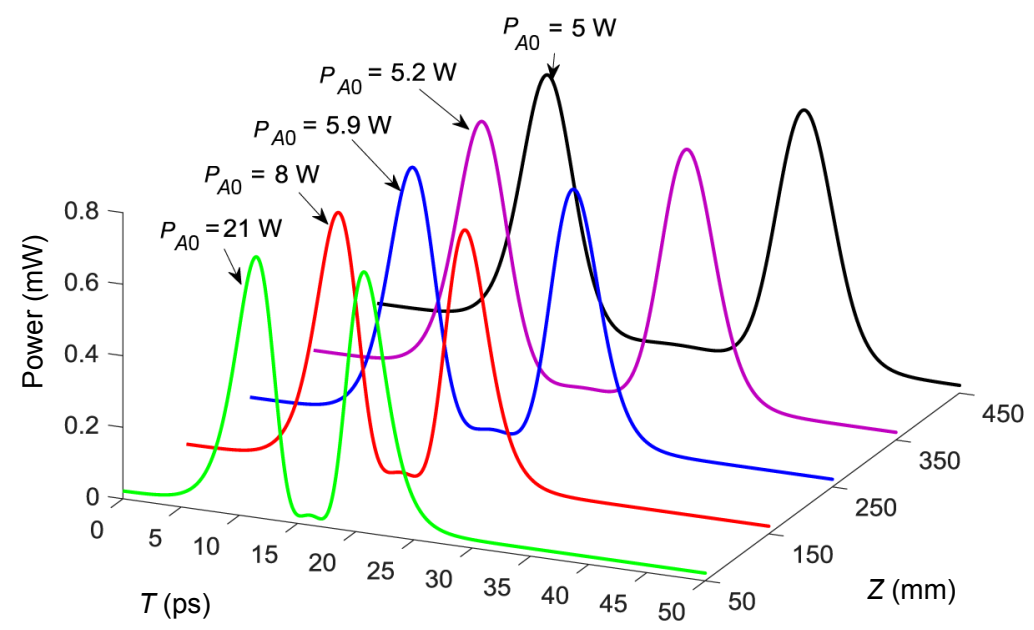

Fig. 6. Temporal shapes of the double pulses with various MOF lengths.

pulse is significantly extended as a result of the influence of the walk-off effect for the longer MOF line, i.e. the corresponding spacing is, respectively, equal to $\sim 9.3, \sim 10.9, \sim 14.2, \sim 17.6$ and $\sim 22.1$ ps for various MOF lengths in this plot.

In the cases of $P_{A 0}=3 \mathrm{~W}$ and $Z=450 \mathrm{~mm}$, the influence of the temporal width of the pulse $A_{0}$ on the outcome pulse is displayed in Fig. 7 , in which the pulse width $T_{A 0}$ is selected at $2,4,6,8$, 10 and 12 ps. Actually, the nonlinear temporal interaction range of two optical fields is in proportion to the initial temporal width of the pulse $A_{0}$ so that the strength of nonlinear interaction between the pulse $A$ and the wave $B$ is gradually enhanced with the increase of the pulse $A_{0}$ width. While the pulse $A_{0}$ width is compressed to $2 \mathrm{ps}$, the single pulse with an about $0.38 \mathrm{~mW}$ peak level is generated, and it can be estimated that the maximum phase difference of two recombined fields is less than $2 \pi$. After the temporal width, $T_{A 0}$, is increased to $4 \mathrm{ps,}$ the peak of the single pulse approaches $\sim 0.72 \mathrm{~mW}$ as a result of the $\sim 2 \pi$ phase difference induced by the enhanced nonlinear interaction of two optical fields. As the corresponding phase difference is up to $2 \pi$, the output pulse is evolved into two peaks and the double pulses, as illustrated at $T_{A 0}=6,8$, 10 and 12 ps. In addition, it is seen that the spacing of the double pulses is extended because of the increase in the temporal width of the pulse $A_{0}$. However, due to the effect of the fully constructive interference at the $2 \pi$ phase difference, the peak of the generated pulse is always kept at a constant of

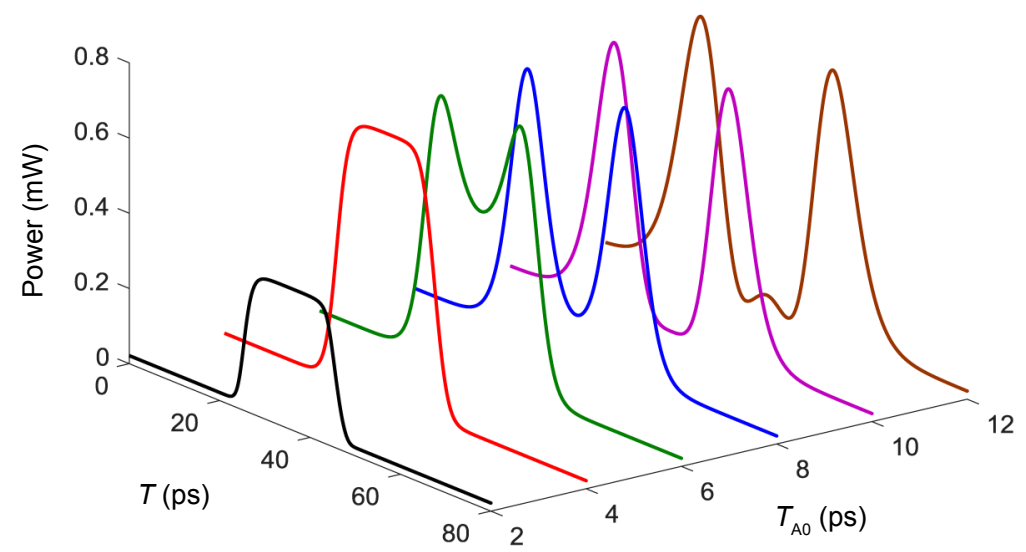

Fig. 7. Temporal shapes of outcome pulses with various widths for the pulse $A_{0}$. 
$\sim 0.72 \mathrm{~mW}$ as the width of the pulse $A_{0}$ is enlarged from 4 to 12 ps.

\section{Conclusions}

In conclusion, an MZI consisting of a microstructured optical fibre with an $\mathrm{AsSe}_{2}$ core is presented to generate single and double pulses. The main physical mechanism for generation of the outcome pulse is attributed to the constructive or destructive interference arising from the phase difference of recombined two optical fields. In the sample arm of the MZI, both the continuous wave $B$ and the pulse $A$ are simultaneously launched into the MOF line with the result that the phase shift of the wave $B$ is mainly controlled by the co-propagating pulse $A_{0}$ with an enough high peak power and temporal width. In the reference arm of the MZI, the wave $B$ phase is slightly shifted due to its SPM. Therefore, a significant phase difference for the recombined optical fields is attained in the output port of the MZI. Resulting from the interference effect, both the single and the double pulses are switchable by selecting various parameters such as the peak power and temporal width of the pulse $A_{0}$, as well as the MOF length that can be properly adjusted to achieve various phase differences. If the phase difference is less than or equal to $2 \pi$, the single pulse with a single peak is generated. One pulse with two peaks and the perfect double pulses can be obtained as the phase difference is varying from 2 to $3 \pi$. By increasing the MOF length and the temporal width of the pulse $A_{0}$, the width of the single pulse and the spacing of the double pulses are significantly broadened.

\section{Acknowledgements}

This work was supported in part by the Open Foundation of the State Key Laboratory on Integrated Optoelectronics under Grant IOSKL2016KF02.

\section{References}

[1] T. Fjelde, D. Wolfson, P.B. Hansen, A. Kloch, C. Janz, A. Coquelin, I. Guillemot, F. Gaborit, F. Poingt, and B. Dagens, 20 Gbit/s optical wavelength conversion in all-active Mach-Zehnder interferometer, Electron. Lett. 35(11), 913-914 (1999).
[2] J.W. Wu and A.K. Sarma, Ultrafast all-optical XOR logic gate based on a symmetrical MachZehnder interferometer employing SOI waveguides, Opt. Commun. 283(14), 2914-2917 (2010).

[3] H.M. Gong, X. Chen, Y.R. Qu, Q. Li, M. Yan, and M. Qiu, Photothermal switching based on silicon Mach-Zehnder interferometer integrated with light absorber, IEEE Photon. J. 8(2), 7802610 (2016).

[4] D. Melati, A. Waqas, Z. Mushtaq, and A. Melloni, Wideband integrated optical delay line based on a continuously tunable Mach-Zehnder interferometer, IEEE J. Sel. Top. Quant. 24(1), 4400108 (2018).

[5] X.J. Yu, D. Bu, X.F. Chen, J.T. Zhang, and S.C. Liu, Lateral stress sensor based on an in-fiber MachZehnder interferometer and Fourier analysis, IEEE Photon. J. 8(2), 6801710 (2016).

[6] H. Vahed, S. Aghazadeh, Amplifier action of a nonlinear Mach-Zehnder interferometer by using of saturable nonlinear arm, Indian J. Phys. 91(5), 569-573 (2017).

[7] R.A. Soref, F. De Leonardis, and V.M.N. Passaro, Reconfigurable optical-microwave filter banks using thermo-optically tuned Bragg MachZehnder devices, Opt. Express 26(12), 1487914893 (2018).

[8] A. Kotb and K.E. Zoiros, Performance analysis of all-optical XOR gate with photonic crystal semiconductor optical amplifier-assisted Mach-Zehnder interferometer at $160 \mathrm{~Gb} / \mathrm{s}$, Opt. Commun. 402, 511-517 (2017).

[9] S.W. Guo and J.W. Wu, Pulse generation and compression using an asymmetrical porous silicon-based Mach-Zehnder interferometer configuration, Pramana J. Phys. 87, 91 (2016).

[10]C. Chen, X. Hou, and J.H. Si, Protein analysis by Mach-Zehnder interferometers with a hybrid plasmonic waveguide with nano-slots, Opt. Express 25(25), 31294-31308 (2017).

[11]J.C. Sales, A.F.G.F. Filho, A.C. Ferreira, J.R.R. Sousa, K.M.V. Avila, D.N.S. Cavalcante, F.T.C.B. Magalhaes, C.S. Sobrinho, P.V.F. Pinto, and G.F. Guimaraes, Mach-Zehnder nonlinear interferometer in photonic crystal fibers with 
nonlinearity profiles, J. Nonlinear Opt. Phys. 24(3), 1550036 (2015).

[12]S. Vyas, T. Tanabe, M. Tiwari, and G. Singh, Ultraflat broadband supercontinuum in highly nonlinear $\mathrm{Ge}_{11.5} \mathrm{As}_{24} \mathrm{Se}_{64.5}$ photonic crystal fibres, Ukr. J. Phys. Opt. 17(3), 132-139 (2016).

[13]T.L. Cheng, Y. Kanou, X.J. Xue, D.H. Deng, M. Matsumoto, T. Misumi, T. Suzuki, and Y. Ohishi, Mid-infrared supercontinuum generation in a novel $\mathrm{AsSe}_{2}-\mathrm{As}_{2} \mathrm{~S}_{5}$ hybrid microstructured optical fiber, Opt. Express 22(19), 2301923025 (2014).

[14]L. Chen, W.Q. Gao, L. Chen, P. Wang, C.Q. Ni, X.C. Chen, Y. Zhou, W. Zhang, J.G. Hu, and M.S. Liao, Numerical study on supercontinuum generation by different optical modes in $\mathrm{AsSe}_{2}-$ $\mathrm{As}_{2} \mathrm{~S}_{5}$ chalcogenide microstructured fiber, Appl. Opt. 57(3), 382-390 (2018).

[15]W.Q. Gao, Q. Xu, X. Li, W. Zhang, J.G. Hu, Y. Li, X.D. Chen, Z.J. Yuan, M.S. Liao, and X. Li, Supercontinuum generation in a step-index chalcogenide fiber with $\mathrm{AsSe}_{2}$ core and $\mathrm{As}_{2} \mathrm{~S}_{5}$ cladding, Jpn. J. Appl. Phys. 55(12), 122201 (2016).

[16]L. Liu, T.L. Cheng, K. Nagasaka, H.T. Tong, G.S. Qin, T. Suzuki, and Y. Ohishi, Coherent mid-infrared supercontinuum generation in allsolid chalcogenide microstructured fibers with all-normal dispersion, Opt. Lett. 41(2), 392-395 (2016).

[17]W.Q. Gao, T.L. Cheng, X.J. Xue, L. Liu, L. Zhang, M.S. Liao, T. Suzuki, and Y. Ohishi, Stimulated Raman scattering in $\mathrm{AsSe}_{2}-\mathrm{As}_{2} \mathrm{~S}_{5}$ chalcogenide microstructured optical fiber with all-solid core, Opt. Express 24(4), 3278-3293 (2016).

[18]Z.B. Tian and D.V. Plant, Picosecond flat-top pulse generation using dual-mode fiber MachZehnder interferometers, Opt. Lett. 36(23), 4542-4544 (2011).

[19]S.L. Pan and J.P. Yao, Switchable UWB pulse generation using a phase modulator and a reconfig- urable asymmetric Mach-Zehnder interferometer, Opt. Lett. 34(2), 160-162 (2009).

[20]V. Moreno, M. Rius, J. Mora, M.A. Muriel, and J. Capmany, Integrable high order UWB pulse photonic generator based on cross phase modulation in a SOA-MZI, Opt. Express 21(19), 2291122917 (2013).

[21]R. Palmer, L. Alloatti, D. Korn, P.C. Schindler, R. Schmogrow, W. Heni, S. Koenig, J. Bolten, T. Wahlbrink, M. Waldow, et al., Silicon-organic hybrid MZI modulator generating OOK, BPSK and 8-ASK signals for up to $84 \mathrm{Gbit} / \mathrm{s}$, IEEE Photon. J. 5(2), 6600907 (2013).

[22]P. Cao, X.F. Hu, J.Y. Wu, L. Zhang, X.H. Jiang, and Y.K. Su, Reconfigurable UWB pulse generation based on a dual-drive Mach-Zehnder modulator, IEEE Photon. J. 6(5), 7903206 (2014).

[23]K. Michailovas, A. Zaukevičius, V. Petrauskiené, V. Smilgevičius, S. Balickas, and A. Michailovas, Sub-20 ps high energy pulses from $1 \mathrm{kHz}$ neodymium-based CPA, Lith. J. Phys. 58(2), 159-169 (2018).

[24] V.P. Veiko, V.N. Lednev, S.M. Pershin, A.A. Samokhvalov, E.B. Yakovlev, I.Yu. Zhitenev, and A.N. Kliushin, Double nanosecond pulses generation in ytterbium fiber laser, Rev. Sci. Instrum. 87, 063114 (2016).

[25]I. Beleckaitè, L. Burakauskas, and R. Adomavičius, Study of surface electric field and photocarrier dynamics in InAs by means of a modified double-pump-pulse terahertz emission method, Lith. J. Phys. 58(1), 116-125 (2018).

[26]R.A. Ganeev, M. Suzuki, S. Yoneya, and H. Kuroda, Application of double femtosecond pulses for plasma harmonic generation, Appl. Phys. Lett. 105, 041111 (2014).

[27]A. Consoli and I. Esquivias, Pulse shortening of gain switched single mode semiconductor lasers using a variable delay interferometer, Opt. Express 20(20), 22481-22489 (2012).

\title{
VIENGUBŲ IR DVIGUBŲ IMPULSŲ GENERAVIMAS NAUDOJANT SIMETRINI MACHO IR CE்NDERIO INTERFEROMETRA SU MIKROSTRUKTŪRUOTAIS CHALKOGENIDŲ ŠVIESOLAIDŽIAIS: SKAITINĖ ANALIZĖ
}

\author{
M.-H. Jin, S.-H. Yang, J.-W. Wu \\ Čongčingo normaliojo universiteto Fizikos ir elektronikos inžinerijos mokykla, Čongčingas, Kinijos Liaudies Respublika
}

